\title{
Gamificação como estratégia para o ensino e aprendizagem de engenharia
}

Cristian Teixeira Marques* ${ }^{*}$ Jéssica Alves Galante ${ }^{\star *}$

\section{Resumo}

O grande desafio deste início de século é a crescente busca por metodologias pedagógicas inovadoras capazes de ultrapassar os limites do treinamento puramente técnico e tradicional. Em meio a tantas técnicas, uma das mais utilizadas é a Gamificação. Nesse sentido buscou-se comparar o ensino de uma disciplina de engenharia, aplicado de duas formas distintas, com e sem gamificação, e medir o impacto na participação, engajamento e aprendizagem dos alunos. Como resultado, é possível de se concluir que os alunos que foram expostos a gamificação apresentaram mais interesse em participar das aulas e também reflexo na medição de desempenho através de notas superiores ao ensino tradicional.

Palavras-chave: gamificação; ensino e aprendizagem; ensino de engenharia.

\section{Introdução}

De acordo com Fadel, Ulbricht, Batista e Vanzin (2014), o termo gamificação compreende a aplicação de elementos de jogos em atividades de não jogos, assim, embora a palavra tenha sido utilizada pela primeira vez em 2010, a gamificação tem sido aplicada há muito tempo nos mais diferentes contextos, porém, pode-se afirmar que é neste atual cenário que está estratégia vem ganhando destaque. A prática de ensino através da utilização de ferramentas didáticas que possibilitem

* Mestre em Engenharia Civil pelo Programa de Pós-Graduação em Engenharia Civil e Ambiental da Universidade de Passo Fundo. Professor e Coordenador do Curso de Engenharia Civil da Faculdade CESURG Marau. E-mail: cristian.marques@hotmail.com

* Engenheira Civil Especialista em Gerenciamento de Obras pela Faculdade Meridional (IMED) e MBA em Marketing pela FGV. Professora dos Cursos de Engenharia Civil, Mecânica, Produção e Administração da Faculdade CESURG Marau. E-mail: jessicagalante@hotmail.com 
a promoção e a problematização dos diversos conteúdos e assim oportunize uma maior motivação bem como a aquisição de novos conhecimentos poderá se tornar um importante recurso para potencializar as qualidades que se espera do sistema de ensino.

Domínguez et al. (2013) destacam que jogos são capazes de promover diferentes contextos lúdicos e imaginários na forma de narrativas, imagens e sons, favorecendo assim o processo de ensino e aprendizagem. Para Zichermann e Cunningham (2011), os dispositivos inseridos nos jogos podem funcionar como um motor motivacional para os indivíduos, contribuindo assim para um maior engajamento destes nos mais variados aspectos e ambientes. Vianna et al. (2013) entendem que o grau de engajamento dos indivíduos no jogo pode ser influenciado pelo nível de dedicação que os mesmos colocam na realização das tarefas. Essa dedicação, muitas vezes pode ser traduzida no cumprimento às missões do jogo que estimulam a imersão dos indivíduos em um ambiente recreativo.

Schmitz, Klemke e Specht (2012) entendem que é possível resumir os diferentes elementos dos jogos em três parcelas: o personagem, a competição e as regras do jogo. Dentro deste contexto está inserida a narrativa por meio da construção de uma história em que essas parcelas poderão atuar de diversos modos. Nesse aspecto, a gamificação pode ser aplicada a diversas atividades em que se objetiva o estímulo às habilidades e comportamentos dos indivíduos. Schmitz, Klemke e Specht (2012) exemplificam que no processo de aprendizagem a gamificação pode contribuir tanto na motivação como no desenvolvimento cognitivo do estudante. Campigotto, Mcewen e Demmans (2013) afirmam que a sua utilização também contribui na criação de um ambiente ímpar de aprendizagem, com a eficácia na retenção da atenção do aluno.

As ferramentas digitais cada vez mais presentes, possibilitam a construção de conhecimento de maneira interativa, contínua e colaborativa, não mais ficando esta prática restrita à sala de aula (ARRUDA, 2013). De acordo com o autor, a tecnologia possibilita a ampliação das práticas e da mediação do ensino. Sendo assim, a partir do conhecimento da necessidade que existe no atual cenário da educação, para que o aluno se torne cada vez mais o protagonista na construção do conhecimento, e o professor assuma o papel de mediador, buscando a qualidade de interação e uma exposição ao conhecimento da forma mais correta possível, a utilização de todas as suas funções cognitivas e a melhor adaptação às exigências do meio, este artigo 
traz como reflexão a seguinte questão: Qual o impacto da utilização da gamificação em sala de aula na participação, engajamento e aprendizagem do aluno ao longo dos diferentes processos de ensino e aprendizagem? Para responder esta pergunta, tem-se por objetivo comparar o ensino de uma mesma disciplina, aplicado de duas formas distintas, ou seja, com e sem gamificação, a partir da observação de 3 aspectos que poderão refletir este impacto: o engajamento (realização das atividades), as notas das avaliações e o percentual de presença nas aulas.

\section{Método}

Considerando os aspectos metodológicos para análise da aplicação da gameficação no processo de ensino e aprendizagem na engenharia, o presente estudo foi desenvolvido por meio de um estudo de caso, onde de acordo com Lima et al. (2012), o uso desta metodologia é adequado quando se pretende investigar o como e o porquê de um conjunto de eventos contemporâneos. Gil (2009) também aponta alguns propósitos dos estudos de caso: (a) explorar situações da vida real cujos limites não estão claramente definidos; (b) preservar o caráter unitário do objeto estudado; (c) descrever a situação do contexto em que está sendo feita uma determinada investigação; (d) formular hipóteses ou desenvolver teorias e (e) explicar as variáveis causais de determinado fenômeno em situações complexas que não permitam o uso de levantamentos e experimentos.

Este artigo realiza uma breve revisão bibliográfica sobre a gamificação, e traz uma análise da comparação do ensino de uma disciplina de engenharia civil, com e sem a aplicação desta ferramenta fazendo uma discussão para melhor entendimento a respeito do impacto da gamificação no ensino e aprendizagem. O estudo foi desenvolvido de forma prática com a disciplina de Tecnologia da Construção de Edificações do curso de Engenharia Civil da Faculdade CESURG Marau, a qual foi ministrada sem aplicação da gamificação em 2019-02, e em seguida aplicada com a utilização da gamificação em 2020-02.

Inicialmente foram observados os resultados obtidos com o engajamento, medido através da realização das atividades propostas em aula, as notas das avaliações e o percentual de presenças dos alunos, para a disciplina aplicada de forma gamificada. Em seguida estes mesmos critérios foram analisados para a disciplina aplicada de forma não gamificada. Por meio de uma comparação entre os resultados encontra- 
dos, buscou-se compreender o quanto esta ferramenta auxiliou ou não na melhora do aprendizado dos alunos.

\section{Resultados e Discussões}

De forma geral, a Gamificação está relacionada ao uso de jogos no processo de ensino e aprendizagem na educação, com o objetivo de promover um maior engajamento e participação dos alunos, e assim tornar as disciplinas mais atraentes e produtivas. Nesse sentido, o presente estudo de caso adotou como estratégia a aplicação da gamificação na disciplina de Tecnologia da Construção de Edificações no curso de Engenharia Civil, baseando-se na ementa da disciplina, que basicamente, abrange o ensino das técnicas e procedimentos de execução de obras na construção civil em todas as suas etapas, desde as fundações até a entrega final da obra. Na sequência pode ser encontrada a discussão dos resultados observados na aplicação da gamificação. A análise foi dividida em 2 tópicos, sendo o primeiro uma discussão acerca da aplicação do jogo e a metodologia utilizada, e em seguida a comparação entre as métricas referentes aos critérios estabelecidos para análise.

\section{Descrição da aplicação da gamificação}

O jogo aplicado a turma de Tecnologia da Construção de Edificações foi esquematizado a partir da construção de uma narrativa onde os alunos foram os personagens principais, ou seja, os engenheiros responsáveis pela execução das obras. Para que esta narrativa pudesse ser apresentada de forma mais atrativa foi elaborado um vídeo por meio da construção de uma história em quadrinhos, como pode ser observado de forma parcial na Figura 1. 
Figura 1: História em Quadrinhos da Narrativa do Game
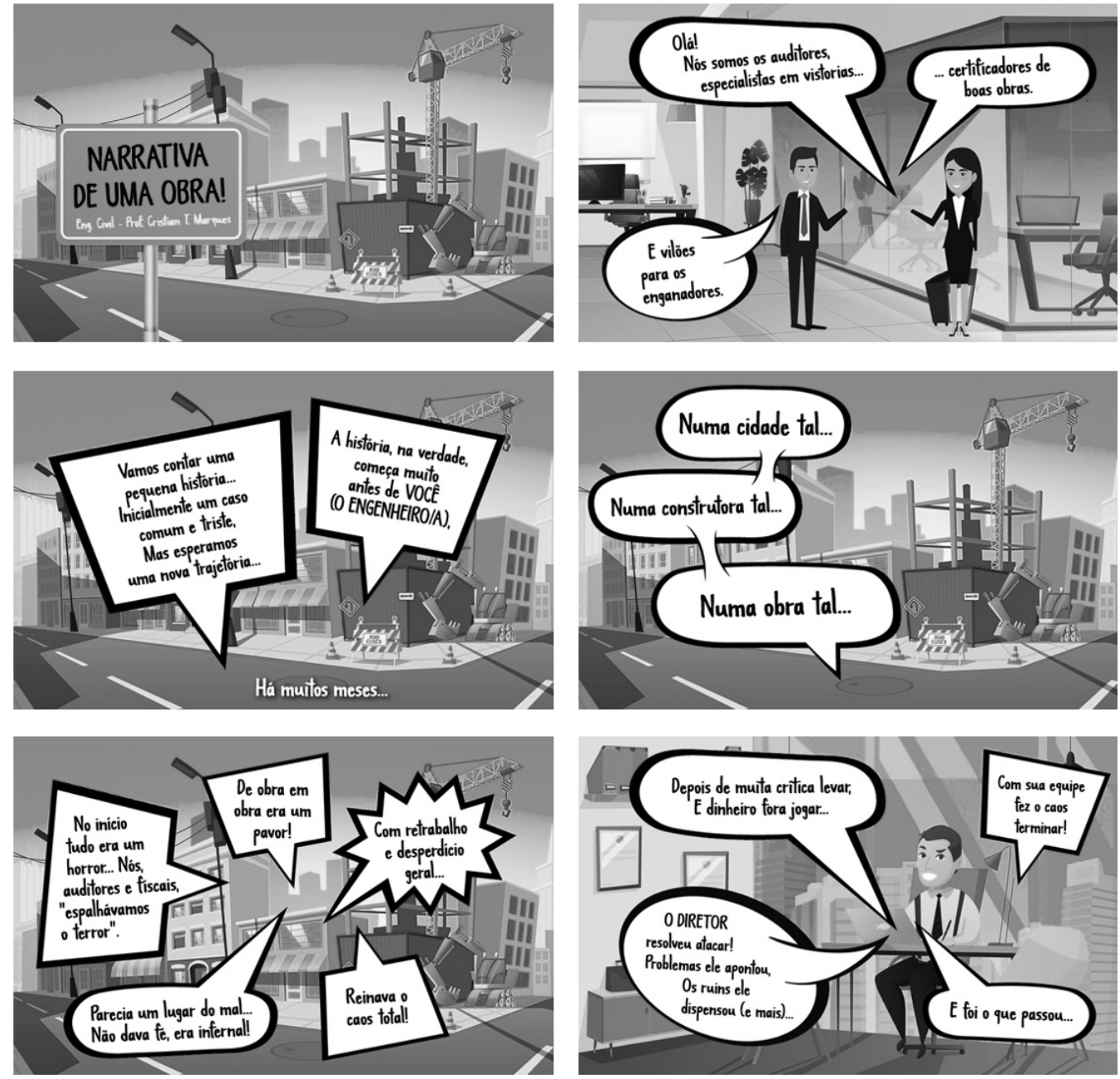

A partir disto, o objetivo do jogo foi fazer com que os alunos cumprissem todas as missões a fim de realizar a construção da obra com qualidade, em todas as suas etapas, de acordo com as boas práticas aprendidas em aula. A cada semana, os alunos foram convidados a cumprir uma nova fase do jogo, a qual sempre correspondia à uma nova etapa da execução da obra, sendo:

- Etapa 1 - Canteiro de Obras;

- Etapa 2 - Infraestrutura;

- Etapa 3 - Superestrutura; 
- Etapa 4 - Estrutura de Concreto Armado;

- Etapa 5 - Alvenaria Estrutural;

- Etapa 6 - Execução de Vedações Alvenaria;

- Etapa 7 - Execução de Revestimentos Argamassados;

- Etapa 8 - Execução de Revestimento Cerâmico;

- Etapa 9 - Fase Especial em Laboratório;

- Etapa 10 - Execução de Outros Revestimentos;

- Etapa 11 - Fase Especial em Laboratório;

- Etapa 12 - Execução de Sistema de Impermeabilização;

- Etapa 13 - Aula Especial em Laboratório;

- Etapa 14 - Execução de Sistema de cobertura;

- Etapa 15 - Entrega de Obra.

Dentro de cada etapa, os alunos tiveram de cumprir diferentes missões a fim de conquistar os pontos necessários e passar de fase. Essa pontuação foi contabilizada por meio da utilização de uma ficha de avaliação que considerava 5 aspectos, ou habilidades, representados como estrelas (veja a Tabela 1).

Tabela 1: Critérios de avaliação do jogo

\begin{tabular}{l|l|l}
\hline 1 & Prazo de entrega & Se o aluno entregou a atividade dentro do prazo \\
\hline 2 & Qualidade de apresentação & Se a atividade entregue teve qualidade \\
\hline 3 & Resultados & Se a atividade foi feita de forma correta \\
\hline 4 & Organização & A forma com que a atividade foi elaborada e entregue \\
\hline 5 & Proatividade & $\begin{array}{l}\text { Se o aluno foi proativo em buscar fazer a atividade bem como } \\
\text { apresentar resultados além do esperado }\end{array}$ \\
\hline
\end{tabular}

O feedback acerca da pontuação, com a ficha de avaliação a qual pode ser visualizada na Figura 2, foi feito semanalmente e de forma individual, onde cada aluno teve conhecimento apenas da sua própria pontuação ao longo de todo o jogo. 
Figura 2: Ficha de Avaliação dos jogadores

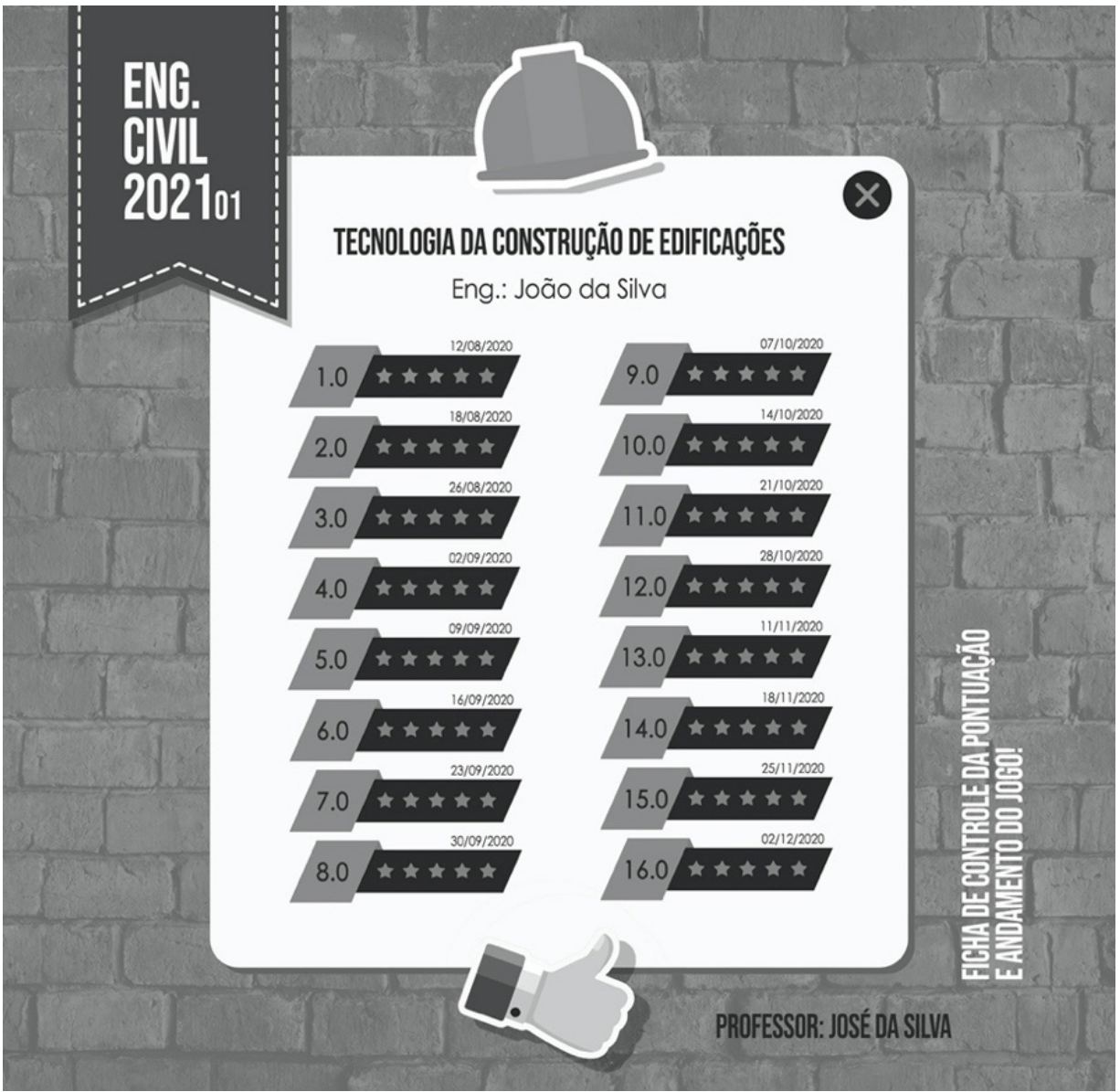

Dentre as missões de cada fase, os alunos tiveram de realizar inúmeras atividades propostas, todas relacionadas ao conteúdo da disciplina, e que foram elaboradas por meio da utilização de outras diferentes ferramentas digitais de ensino e práticas ativas de aprendizagem como Kahoot, Wheel Decide, Google Jamboard, Google Forms, entre outras.

Como forma de engajar ainda mais os alunos, horas antes da aula foram feitas chamadas de divulgação do conteúdo da aula e das missões do jogo do dia, no grupo de comunicação da turma, constatando-se assim de forma frequente o entusiasmo dos alunos. Ao longo de todo o andamento do game foi possível de observar um forte engajamento de todos os envolvidos. Em casos de necessidade de falta por parte alu- 
nos, por exemplo, as mesmas sempre foram justificadas, e ainda nestes casos, todos buscaram junto ao professor possíveis formas de efetuar a recuperação da pontuação.

\section{Comparação dos resultados de engajamento e aprendizagem}

Dentre as disciplinas analisadas neste estudo, ambas as turmas são de Tecnologia da Construção e Edificação, ministradas no sexto semestre da matriz 31 do Curso de Engenharia Civil. Uma foi ministrada com a utilização da gamificação, metodologia ativa, aplicada no segundo semestre de 2020, com 9 alunos matriculados, sendo destes 7 com idade abaixo de 30 anos, e outros 2 alunos com idade acima de 30 anos. A outra foi ministrada de forma tradicional e aulas presenciais, no segundo semestre do ano de 2019, com 7 alunos matriculados, sendo destes 6 com idade abaixo de 30 anos, e 1 aluno com idade acima de 30 anos (Figura 3).

Figure 3: Caracterização da amostra
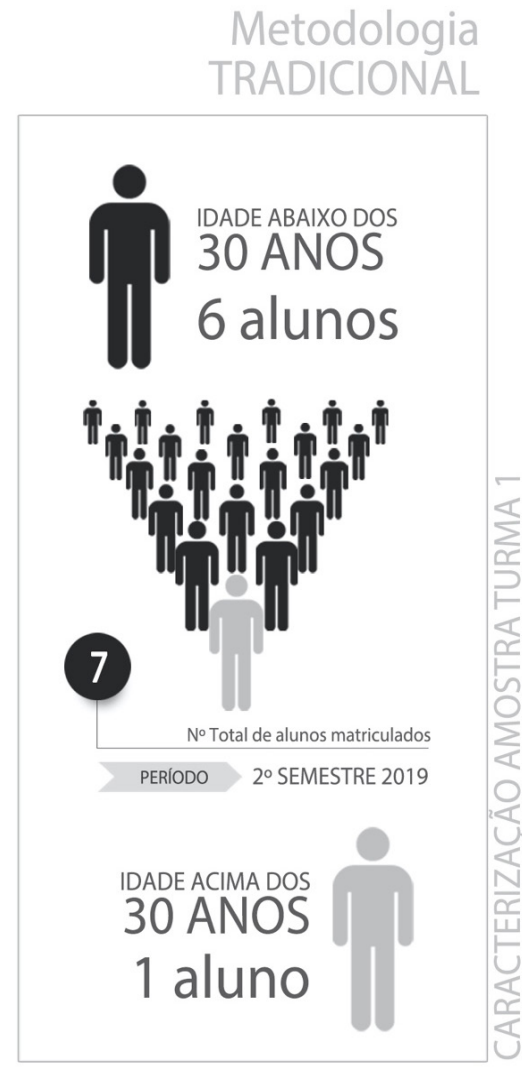

Metodologia com GAMIFICAÇÃO

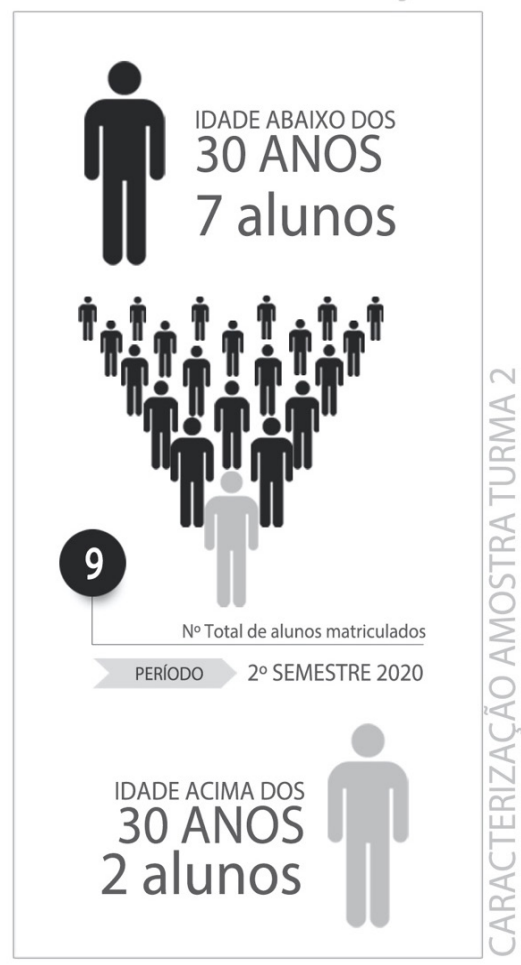


Vale ressaltar que a disciplina ministrada de forma gamificada ocorreu durante o período de pandemia do COVID-19, com todas as aulas teóricas ministradas em formato on-line, através do Google Meet, e as aulas práticas de forma presencial nos laboratórios da faculdade, sempre respeitando as orientações e bandeiras do Estado do Rio Grande do Sul.

Para realizar a análise dos dados e resultados obtidos na comparação entre a metodologia tradicional e a metodologia ativa, através do uso da gamificação, foram definidos três critérios considerados relevantes para a análise: (a) frequência nas aulas; (b) entrega de atividades; e (c) notas das avaliações.

Em uma primeira análise, considerando o primeiro critério definido, é possível de perceber um aumento de $86 \%$ na frequência de 2019 , onde foi utilizada metodologia tradicional presencial, para 93\% em 2020, com gamificação no sistema híbrido, representando um aumento de 7\% entre eles. Na segunda análise, considerando o segundo critério no item que corresponde a entrega de atividades, a disciplina de 2019 apresentou uma devolutiva média de $88 \%$ das atividades, e no ano de 2020 o resultado foi um acréscimo de $4 \%$, chegando a $92 \%$ das atividades entregues. $\mathrm{Na}$ terceira análise, levando-se em conta o terceiro critério, que foram as notas das avaliações, é possível de observar uma representatividade muito maior nas mudanças e desempenho dos alunos, pois enquanto que em 2019, 50\% da turma necessitou de prova complementar de nota, ou seja, prova de exame, para ser aprovado na disciplina, em 2020 com a gamificação todos os alunos alcançaram a média sem necessidade de prova suplementar, ou seja, todos foram aprovados com as avaliações regulares da disciplina. Além disso, a média geral nas notas subiu de 7,55 para 8,62 com uso da metodologia ativa, onde em percentual esse aumento representa aproximadamente $15 \%$ entre as médias.

Para representação em estalas centesimais de todos os parâmetros utilizados na análise, foi necessário multiplicar por 10 as notas dos alunos, demonstrando a representatividade do crescimento em aprendizagem e comprometimento entre os dois métodos analisados, representados na Figura 4 representada pelo gráfico de desempenho em notas, entregas e frequência dos alunos das duas turmas e metodologias avaliadas. 
Figure 4: Gráfico de comparação dos resultados

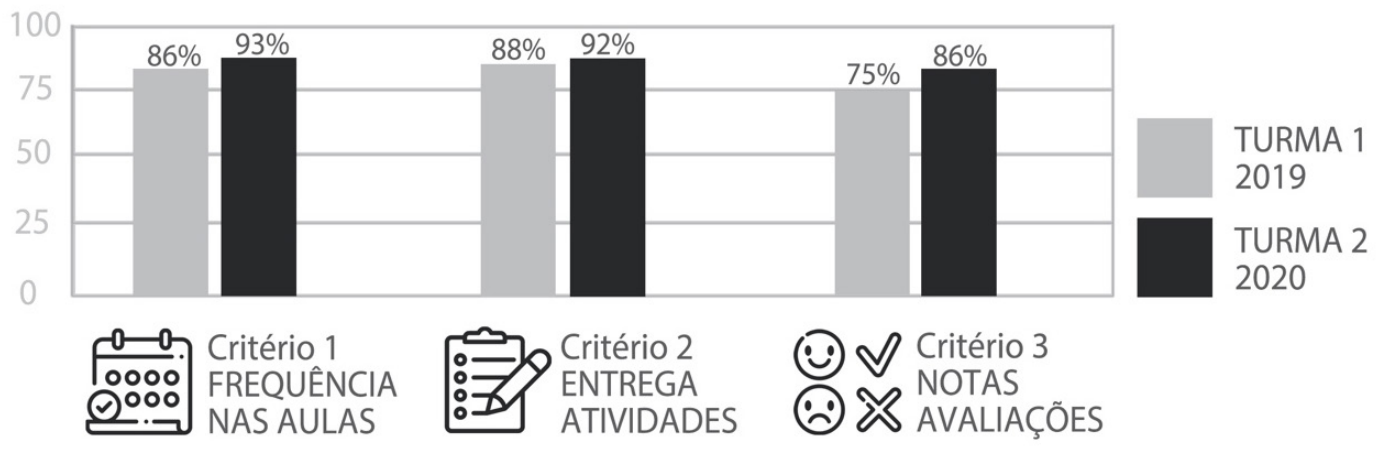

Todos esses resultados esquematizados no gráfico da Figura 4, auxiliam para uma melhor compreensão das diferenças de desempenho constatadas da sua observação e dos dados obtidos a partir da medição dos critérios definidos para análise.

\section{Considerações}

Diante de todas as análises, é possível de se concluir que os alunos que foram expostos a gamificação apresentaram um maior interesse em participar das aulas, e isto se refletiu na medição de desempenho deles através das notas de avaliações superiores às observadas no ensino tradicional. Desta forma, pode-se comprovar por meio da comparação entre ambas as turmas, que a utilização da gamificação em sala de aula propiciou um impacto positivo relevante na aprendizagem dos alunos.

Apesar das amostras analisadas serem pequenas, as características entre elas são muito semelhantes, em função da idade, da disciplina no mesmo semestre da matriz curricular. Desta forma, entende-se que o conhecimento prévio de ambas as turmas foram os mesmos, o professor responsável por ministrar as disciplinas em ambas as metodologias foi o mesmo, ementas foram as mesmas, e a diferença do período de aplicação entre uma e outra foi de apenas de 1 ano.

Em função de todo o período da aplicação desta metodologia ativa ter sido desenvolvido em meio a pandemia, se considera ainda mais relevante o estudo comparativo com ensinos ministrados de formas iguais, sendo eles, presenciais, online ou híbrido, pois esta mudança de comportamento, desempenho e frequência dos alunos perante a forma de ensino pode ser ainda mais incentivadora e representativa. 


\section{Gamification as a strategy for engineering teaching and learning}

\section{Abstract}

The great challenge of the beginning of this century is the growing search for innovative pedagogical methodologies capable of exceeding the limits of purely technical and traditional training. Among so many techniques, one of the most used is Gamification. In this sense, we sought to compare the teaching of an engineering discipline, applied in two different ways, with and without gamification, and to measure the impact on student participation, engagement and learning. As a result, it is possible to conclude that students who were exposed to gamification were more interested in participating in classes and also reflected in the performance measurement through grades higher than traditional teaching.

Keywords: gamification; teaching and learning; engineering education.

\section{Referências}

ARRUDA, E. P. Ensino e aprendizagem na sociedade do entretenimento: desafios para a formação docente. Revista Educação PUC-RS, v. 36, n. 2, p. 232-239, 2013. Acesso em agosto de 2021, disponível em: file:///Users/admin/Downloads/12036-Texto\%20do\%20artigo-54534-2-10-20130702.pdf

CAMPIGOTTO, Rachelle; McEWEN, Rhonda; DEMMANS, Carrie. Especially social: Exploring the use of an iOS application in special needs classrooms. Journal Computers \& Education, Virginia, v. 60, p. 74-86, 2013. Acesso em agosto de 2021, disponível em: https://eric.ed.gov/?id=EJ1006984

DOMÍNGUEZ, Adrián; NAVARRETE, Joseba Saenz de; MARCOS, Luis de; SANZ, Luis Fernández; PAGÉS, Carmen; HERRÁIZ, José Javier Martínez. Gamifying learning experiences: Practical implications and outcomes. Journal Computers \& Education, Virginia, v. 63, p. 380-392, 2013. Acesso em agosto de 2021, disponível em: https://doi.org/10.1016/j.compedu.2012.12.020

FADEL, Luciane Maria; ULBRICHT, Vania Ribas; BATISTA, Claudia Regina; VANZIN, Tarcísio (org.). Gamificação na educação. São Paulo: Pimenta Cultural, 2014. 300 p. Acesso em agosto de 2021, disponível em: http://www.pgcl.uenf.br/arquivos/gamificacao_na_educacao_011120181605.pdf

LIMA, João Paulo Cavalcante et al. Estudos de Caso e sua aplicação: Proposta de um esquema teórico para pesquisas no campo da contabilidade. Revista de Contabilidade e Organizações, São Paulo, v. 6, n. 14, p.127-144, 2012. Acesso em agosto de 2021, disponível em: https://www.redalyc. org/pdf/2352/235223852007.pdf

SCHMITZ, Birgit; KLEMKE, Roland; SPECHT, Marcus. Effects of mobile gaming patterns on learning outcomes: a literature review. Journal Technology Enhanced Learning, 2012. Acesso em agosto de 2021, disponível em: 10.1504/IJTEL.2012.051817

VIANNA, Ysmar; VIANNA, Maurício; MEDINA, Bruno; TANAKA, Samara. Gamification, Inc.: como reinventar empresas a partir de jogos. MJV Press: Rio de Janeiro, 2013. Acesso em agosto de 2021, disponível em: http://www.gestaoporprocessos.com.br/wp-content/uploads/2014/06/Gamification-Inc-MJV.pdf

ZICHERMANN, Gabe; CUNNINGHAM, Christopher. Gamification by Design: Implementing Game Mechanics in Web and Mobile Apps. Sebastopol, CA: O’Reilly Media, Inc. 2011. Acesso em agosto de 2021, disponível em: http://storage.libre.life/Gamification_by_Design.pdf 